\title{
The Meaning of Genetic Research Results: Reflections from Individuals With and Without a Known Genetic Disorder
}

\author{
R. Jean Cadigan, \\ University of North Carolina \\ Marsha Michie, \\ University of North Carolina \\ Gail Henderson, \\ University of North Carolina
}

Arlene M. Davis, and

University of North Carolina

Laura M. Beskow

Duke University

\section{Abstract}

In the debate about whether to return individual genetic results to research participants, consideration of the nature of results has taken precedence over contextual factors associated with different study designs and populations. We conducted in-depth interviews with 24 individuals who participated in a genotype-driven study of cystic fibrosis: 9 of the individuals had cystic fibrosis, 15 had participated as healthy volunteers, and all had gene variants of interest to the researchers. These interviews revealed that the two groups had different ideas about the meaningfulness of genetic results. Our findings point to the importance of understanding research context, such as participants' relationship with the researcher and whether they have the disease condition under study, when considering whether to return individual results.

\section{Keywords}

cystic fibrosis; healthy volunteers; biobank; genetic research; qualitative data; return of results

In the field of human genetic research, much debate has been generated concerning the return of individual results to research participants. Recommendations on return of results have been issued by the National Heart, Lung, and Blood Institute and the National Bioethics Advisory Commission (Bookman et al., 2006; Fabsitz et al., 2010; NBAC, 1999), and the topic addressed by many respected bioethicists, clinicians, and scientists. Although this debate has highlighted a number of important features related to the research results themselves, few have also taken into consideration factors associated with different study designs and study populations (Beskow \& Burke, 2010; Hoeyer, 2010). For example, researchers may have different responsibilities toward or choose to share different kinds of information with participants who have been diagnosed with a serious condition, or participants with whom they have an ongoing relationship or interactions (Beskow \& Burke, 
2010). As researchers and ethics boards are increasingly called upon to make case-by-case decisions about whether and how to return different kinds of results, they will likely need to consider a more nuanced range of factors beyond the nature of the results themselves.

Genotype-driven research recruitment presents particular challenges with regard to return of results. Such recruitment is driven by genotypic rather than phenotypic information about an individual. The prospective participant often has no prior knowledge of the genetic information of interest, which is typically a research-generated finding from a prior study. Thus, researchers must decide whether to disclose the genetic inclusion criteria that make individuals eligible when recruiting them for the follow-up study.

The research presented below was part of a larger three-site study exploring participants' perspectives on genotype-driven research recruitment (Beskow et al., 2011). Specifically, we interviewed two groups of research participants (patients with cystic fibrosis, selected from a prior genetic study, and healthy volunteers, selected from a genetic biobank) who took part in a genotype-based cystic fibrosis study, and who were both informed, as part of recruitment, that they had one of two genetic variants under study. In this paper, we examine the different ways that these two groups conceptualized genetic research results as meaningful, and, in turn, the ways that their preferences for receiving those results differed.

Recommendations on the return of genetic research results related to serious medical conditions have usually considered two main characteristics of the results: validity and utility (Bredenoord, 2011; Dressler, 2009). The validity of results refers not only to analytic validity - the certainty with which researchers identify a particular genetic trait in a subject; but also to clinical validity - the strength of that trait's connection with a clinical outcome. Utility refers to the usefulness of those results. Results may have clinical utility (useful to inform risk reduction strategies or to improve health outcomes), personal utility (useful for personal or familial reasons, such as retirement planning or other future decision-making), personal meaning (the value of knowledge for its own sake), or some combination of the three (Beskow \& Burke, 2010). All of these considerations are important when making decisions about the return of results in biomedical research, and the meanings of all of these have been contested (Bredenoord, 2011). Indeed, the very definition of "research results" is the subject of debate (Miller et al., 2008), along with what constitutes a "meaningful result" for patients (Miller, Hayeems, \& Bytautas, 2010; Trinidad et al., 2011). In the case of genetic research, decisions may be even more complex, as genetic results may reveal unexpected or unwanted information about family members (Bredenoord, 2011; Dressler, 2009).

In a recent commentary, Beskow and Burke (2010) note that research populations may have differing levels of vulnerability in terms of their health status; differing depths of relationship with ongoing research or particular researchers; and differing degrees of dependence on research for access to genetic information. These characteristics, they argue, shape the context of research and should be taken into account when considering the return of genetic results. In addition, recent reports have documented participants' stated desire for the return of individual results (Murphy et al., 2008; Fisher, 2008; Miller, Hayeems, \& Bytautas, 2010; Michie et al., 2011), or even an expectation that researchers are obliged to share important findings with them (Cadigan \& Davis, 2009). This study offers an opportunity to compare the experiences and perspectives of two research populations who had been given identical information during genotype-driven research recruitment for the same study, yet conceptualized genetic research results differently in light of their very different contexts. 


\section{Methods}

For this qualitative study of participants' perspectives on genotype-driven recruitment, interviews were conducted with individuals who had participated in a study about circulating levels of the protein TGF $\beta 1$ (see Figure 1). Individuals were selected for this study based on the presence of particular genetic traits suspected to play a role in circulating blood levels of TGF $\beta 1$, which in turn have been shown to affect lung function in individuals with cystic fibrosis (Cutting, 2010; Drumm et al., 2005). Half of the TGF $\beta 1$ study participants were individuals with cystic fibrosis who had participated in an earlier study of genetic modifiers of cystic fibrosis headed by the same investigator. Most of these participants had been with the same doctor or team of doctors for much of their lives, and virtually all were routinely asked to participate in research studies in the clinic. The other half of the TGF $\beta 1$ participants were individuals without cystic fibrosis who were recruited from a genetic biobank, the Environmental Polymorphisms Registry (EPR), which serves as a resource for researchers seeking to study particular genetic traits (Chulada et al., 2008).

For our qualitative interviews, we recruited participants with and without cystic fibrosis from the TGF $\beta 1$ ("followup") study. Principal investigators from the "original" studies (the cystic fibrosis genetic modifiers study and the genetic biobank) sent letters on our behalf to participants from each study who had also participated in the TGF $\beta 1$ study. Response cards and stamped envelopes were included so that interested individuals could contact us directly. All interviews were conducted in person at a location of the respondent's choosing, with the exception of one interview that was conducted by telephone because the respondent lived over 60 miles from the study site. Prior to the start of the recorded interview, all participants provided verbal consent to participate. Cadigan and Michie conducted all of the interviews.

This study was determined exempt by the Duke University Health System (DUHS) Institutional Review Board (IRB), the institution at which this multi-site study was based. The IRB at The University of North Carolina at Chapel Hill (the site responsible for recruiting the above participants and conducting interviews) agreed to rely on the DUHS IRB's decisions.

\section{Genotype-Driven Recruitment Process for the TGF $\beta 1$ Study}

Cystic fibrosis (CF) participants were recruited for the TGF $\beta 1$ study at a clinical visit, during which a researcher explained the study to them. Biobank participants were asked to join the TGF $\beta 1$ study via a letter that outlined the goals of the study and explained that recipients were being recruited because of a trait in their DNA; however, the letter did not provide any more specific genetic information.

The consent form, which was identical for CF participants and biobank participants, briefly explained the TGF $\beta 1$ protein and previous research related to genes and TGF $\beta 1$. It stated, "Previous studies have shown that in cystic fibrosis (CF) genotypes called CC and TT are related to different levels of TGF $\beta 1$ and how severe the disease is." The consent form then offered categorical genetic inclusion criteria for study participants (Box 1).

\section{Box 1}

\section{Genetic Inclusion Criteria for TGF $\beta 1$ Study}

You are being asked to be in the study because you are either:

1 A cystic fibrosis patient who consented to donate blood for research as part of the Gene Modifier Study. During this study, your blood was tested to 


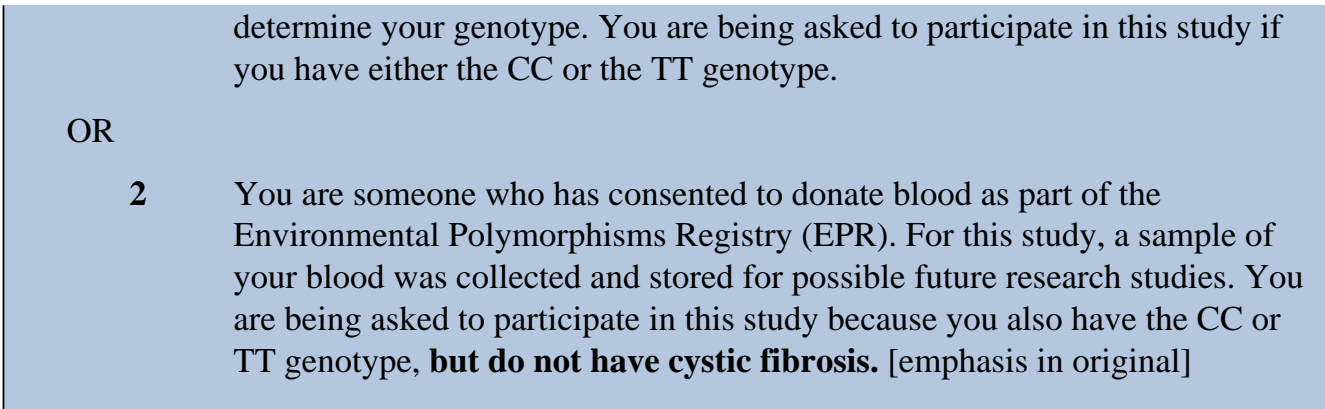

During the first few interviews it became clear that virtually none of the participants recalled reading this passage, and that most did not understand that they were asked to join the TGF $\beta 1$ study because a previous study had identified a particular trait in their genotype. Hence, for a subset of the interviews, we added a brief interaction at the end of the protocol in which we showed participants a consent form identical to the one they signed for the TGF $\beta 1$ study, highlighting the above passage that explained the genetic inclusion criteria. Interviewers asked participants to read this passage, discussed their reactions to it, and asked them to revisit some of the earlier interview topics with this information in mind.

\section{Interview Topics}

Interviewees were asked to recall their experiences of both the original study (either the cystic fibrosis genetic modifiers study or joining the biobank) and the followup study (the TGF $\beta 1$ study), and provided opinions on the return of individual genetic research results in the context of recontact for a genotype-driven follow-up study (see Beskow et al., 2011, for more detailed information about the interview protocol). The analysis presented here focuses on interviewees' comments about the return of individual genetic research results in the context of genotype-driven recruitment. Although participants had been told only that they had one of two genetic traits (not which one) during recruitment and the consent process for the TGF $\beta 1$ study, and rarely recalled receiving any genetic information at all, the topic of individual results was a rich one in our interviews. All respondents provided comments about the nature of individual results and the circumstances under which they should be offered. These discussions usually occurred in response to a set of questions on these topics, but also sometimes arose spontaneously during other parts of the interview. At the beginning of this set of questions, interviewees were read a definition of individual genetic research results (Box 2) in order to ensure they were given identical information.

\section{Box 2}

\section{Definition of Individual Genetic Research Results}

Researchers doing a genetic study are usually trying to find out whether there is a link between a genetic trait and the medical condition they are studying. By looking at the DNA from everyone in the study, researchers are sometimes able to find such a trait. Some people in the study have that particular trait and some people don't. Also, researchers sometimes know something about that genetic trait and what it might meanbut often they are not sure what it means or if it is even related to the medical condition until they do more studies. So, when I say, "individual genetic research results," I mean information about whether or not you have the genetic trait that researchers identified in their study. 


\section{Analysis}

All interview recordings were transcribed and identifying references redacted. Transcripts were imported into QSR International's NVivo 8 qualitative data analysis software. Through a collaborative process with the other two sites in the larger study (Beskow et al., 2011), a codebook was created. All transcripts from UNC's site interviews were coded by Cadigan and Michie using this codebook. Twenty percent of the transcripts were coded by both coders and any discrepancies were resolved by consensus (Miles \& Huberman, 1994).

Cadigan and Michie then examined more closely the responses to the series of interview questions concerning individual research results. They read these interview segments aloud to one another, noting similarities and differences between these responses. Similarly, these segments were compared to the interview addendum in which 14 interviewees were asked to read and respond to the section of the TGF $\beta 1$ consent form. From these detailed examinations, they compiled a list of the characterizations interviewees provided of individual research results, and a list of circumstances under which these participants said that individual research results should or should not be returned to study participants. These two lists were developed in an iterative fashion as new segments were read and analyzed and compared with previous read segments (Charmaz, 2006). The resulting categories were then compared against text related to the nature of individual results or of preferences for return of results, as coded in NVivo.

\section{Results}

All participants in our interview study were White and non-Hispanic, reflecting the racial and ethnic composition of the TGF $\beta 1$ study population. Overall, the respondents were welleducated (Table 1), particularly the biobank participants. The biobank's original recruitment base included regional universities and research-related entities; thus, several of the biobank participants were themselves scientific researchers or physicians, or had previously worked in scientific research positions.

\section{How Meaningful Are Individual Genetic Research Results?}

Though our interview protocol did not specifically ask respondents about their perceptions of meaningfulness of individual genetic research results, our analysis of the interviews indicates that both biobank and $\mathrm{CF}$ participants assumed a degree of meaningfulness not necessarily implied by the text of the definition we offered (Box 2). The two groups differed regarding the meanings they offered for research results, although both groups invoked validity and/or utility.

Biobank Participants-Biobank participants' perceptions of meaningfulness of individual results often included an assumption of truth or "real knowledge," and an assumption that the results would contain "bad news" or negative information.

"Real Knowledge": Despite our definition of individual genetic research results emphasizing that researchers often are not sure what results mean or if they are related to the medical condition under study, many biobank participants assumed that individual genetic results would offer conclusive "truth" and that researchers would understand the implications of the result. For example, one biobank participant remarked:

Why would an investigator not communicate the results? [Interviewer gives example of researchers not knowing what results mean yet.] But I was assuming that they knew. That their data was definitive. If the data's not conclusive, you shouldn't share that with anybody. (C19) 
Similar to this participant, many respondents' assumptions regarding the certainty of results were brought into focus when they were specifically asked about uncertain results—and this typically led to a negative opinion about whether such results ought to be returned. When asked if researchers should offer results that have uncertain meaning, another biobank participant responded, "No. Those are not real knowledge. It's not truth yet. It's a speculation. It's a hypothesis. It's a guess. And I don't think that's fair to put that in somebody's head" (C09).

"Bad News": In addition to assuming that research results would offer "real knowledge," many biobank participants believed that genetic results would convey bad news. Two respondents (C18 and C22) likened the return of results to opening a "Pandora's box." While some of our interview questions about return of research results referenced respondents' actual experiences of being recruited for the follow-up study, some asked instead for general opinions. In these latter responses, biobank participants' talk of results expanded well beyond discussion of cystic fibrosis; they brought up many other serious medical conditions, such as various forms of cancer, Alzheimer's disease, and amyotrophic lateral sclerosis (Lou Gehrig's disease). Because of their belief that results offered "real knowledge," biobank participants often assumed that research results would be valid, robust, and predictive, indicating their risk of developing a serious medical condition or passing that condition to their biological children. Almost no one talked about the possibility that research results might convey good news, such as the presence of a protective genetic trait. Thus, when asked what results might be beneficial to learn, respondents still responded in the negative:

...Anything that was abnormal or red flagged on my DNA. Anything that kind of stood out as like this either is a problem, could potentially be a problem, could be passed on to my children or anything like that. ... that would be beneficial for me now or later that I might need to go seek medical help with or something like that or start watching my diet or something like that. That would be important for me to know. (C12)

Many respondents, like this one, linked the benefit of receiving results to the assumption that results offer negative, but definitive knowledge. In addition, this respondent went a step further in assuming utility: she surmised she would be able to get treatment or alter her lifestyle or know in advance her children's risk of certain medical conditions.

Some biobank participants seemed to assume that not receiving individual genetic research results would mean that they are "OK." If results are bad news, then "no news is good news." One respondent, when asked, "How did you feel about not getting individual genetic research results [when you were contacted about the follow-up study]?" commented:

I think I just kind of assumed that that means I don't have [abnormal results]. You know, no news is good news. So it's the fact that I wasn't contacted. It's kind of like when you go and get like a strep test at the doctor, and they're like, "We'll call you if you have strep. If you don't hear from us, you don't." Kind of like that. Like if I don't receive any sort of information, I'm good. (C12)

This respondent, like some others, discussed research results as if they were akin to clinical test results. Moreover, in response to the question, "How did not getting individual genetic research results from the first study affect your decision to be in the second study?," she noted, "It didn't because I figured I was clear" (C12).

Thus many biobank participants, who did not have the condition under study and who (as part of eligibility criteria for the follow-up study) had no other chronic health conditions, assumed that results would offer real knowledge with definite, negative implications. 
Moreover, for some, not receiving results also had meaning because it indicated that one's DNA was "clear" or otherwise good.

Cystic Fibrosis Participants-CF participants' conceptions of individual genetic research results were similar to those of biobank participants in that they, too, assumed meaningfulness of results that was related to validity. However, their responses more often also invoked utility. CF participants had more flexible standards than biobank participants for what they would consider useful. These standards focused on the presumption that results would offer them additional information about their condition, even in the absence of immediate clinical utility. Unlike biobank participants who conceptualized results to be about all kinds of possible severe medical conditions, CF participants largely confined their talk of individual results to results about cystic fibrosis. They strongly believed in both the promise of research to improve lives for those with cystic fibrosis and the idea that individual research results would tell them more about their own bodies and condition.

The Promise of Research: In contrast to biobank participants' assumption that research would produce results that would be "bad news," CF participants tended to talk about the promise of research. They believed research would lead to a cure or improved treatments and therapies for themselves and the "CF community." One respondent, when asked why he participated in the genetic modifiers parent study, commented, "They just came and asked me if I wanted to participate, and I said 'Sure. Anything I can do to help out.' I mean, finding the cure maybe for cystic fibrosis or helping out with the genes and stuff " (C02). In fact, some respondents seemed particularly hopeful about genetic studies of cystic fibrosis. One respondent remarked:

Any time I'm asked to do a study that focuses on genetic stuff, I'm kind of inclined to participate because I still kind of like the pie-in-the-sky dream of "Oh, maybe one day we can have a drug where it just fixes everything." Instead of, "Oh, we're going to treat this little part of your disease, and then we're going to treat this little part, and you're going to have a million different things to keep track of." So even though I don't think right now they're working as much on a blanket "we're going to re-do all your genes" cure, if I can do anything that helps with something remotely related to that, then I'm inclined to participate. (C06)

Some respondents specifically tied the promise of research to receiving research results. As one participant noted when asked why researchers should offer individual results:

I just think it's important for people to know. ... I like to know because if there's gonna be a new drug to market or something that's gonna be beneficial to me, that's something that I'd want to know. You know, and if it's not gonna benefit me, then I'd like to know that, too. (C13)

For this participant, even results with uncertain meaning held promise and were desirable for that reason:

Well, for me I just like to know that kind of information. Changes, any new developments, anything that would further another study that I might be able to get involved in where they could just look into it a little bit further. (C13)

As was the case for many participants with cystic fibrosis, this respondent saw the possibility of ongoing research participation as part of the road to better treatments for himself and the entire CF community.

"This is my body and I want to know": Most CF participants stated that they wanted individual results. As one participant commented, "This is my body and I want to know" 
(C01). These participants believed that individual results would reveal information about their bodies, which would be beneficial even if the implications were bad. Some noted they had already been given the "bad news" that they have cystic fibrosis; more information could only help to deal with the bad news. As one respondent commented, "I mean it might be a little depressing [to get unpleasant individual results] ... but people know they have CF. It's not new" (C06).

Another respondent talked about how she felt pleased to receive an individual result from a prior research study: "It's just more of me taking charge of my CF, you know, knowing about it... It makes you a part of the process" (C01). For CF participants then, individual research results offered the opportunity to understand more about their condition which, in addition to other benefits, was viewed as empowering.

In summary, for CF participants, the meaning of individual research results was different than for biobank participants. They were interested in individual results because they wanted information about their bodies and their disease. Like biobank participants, they often assumed that results would be clinically valid and utilizable. However, having a chronic condition and believing in the promise of research to help ameliorate that condition meant they were more comfortable with uncertain research results.

\section{When Should Individual Genetic Research Results Be Given?}

Because biobank participants and CF participants had different conceptions of the meaningfulness of results, they also had different ideas about the circumstances under which individual research results should be offered to participants.

Biobank Participants-When weighing the risks and benefits of offering results, biobank participants' most frequently mentioned risk was worry. Several used the term "freak out" when discussing how people might react to receiving results. Their concern over people "freaking out" seemed to follow logically from their assumption that results would be clinically valid and convey bad news, particularly in the absence of clinical or other utility:

If it's not actionable, then I don't see any benefit in telling people. ... telling people when there's nothing that they can do about it just doesn't really help. [Interviewer: Do you see specific problems that might arise?] Freaking people out. You know, distress. (C05)

However, many biobank participants assumed that results would in fact be associated with immediate or near-term utility, which balanced their concerns about the risk of distress. When asked what kind of individual results would cause her concern to learn from a researcher, one respondent remarked:

Something that I absolutely cannot change.... If you find out that you have something that's terminal or something that there's nothing out there in terms of medication or help or anything, that's bad news, and to get that kind of bad news is disturbing, but I feel it's their obligation to tell you if that stuff pops up because then you need to find an intervention to help you. (C12)

This respondent, in working through her answer to the interview question, assumed that the bad news conveyed by individual research results could be mitigated by a beneficial intervention and used this assumption to argue that researchers should offer results.

Thus, biobank participants felt more comfortable with researchers offering results that have utility. To some, utility meant, quite broadly, that they could do something with the results. In fact, a few participants argued that there was no such thing as no utility; that is, there is always personal utility. They reasoned that, even without clinical intervention, they might be 
able to marshal resources-by changing their lifestyle or making different life decisionsafter receiving these (presumably bad and definitive) results:

There's no such thing to me as untreatable. ... [K]nowing ten years from now I may have this disease you can make plenty of plans. ... I might want to make sure that I have all my plans in order, health care proxy taken care of, economic lined up. I might want to get- what do they call it? The insurance that you can go into a nursing home. Long-term care. ... Well, and you know particularly from a genetic standpoint you'd want to know. The kids would want to know, and I'd want the kids to know. If it's genetically passed, you have a risk. ... So even if it's incurable, I think it's of value. (C19)

Another participant answered, when asked what the benefits were of offering individual results that lacked utility, "Just so the person would be able to plan their life appropriately" (C22). Though acknowledging that some may suffer anxiety from getting such results, he commented that the good of being able to plan for the future "outweighs the bad" (C22).

Thus biobank participants viewed disclosure of research results as appropriate whenever utility - in the form of available medical treatments or personal actions-outweighed the worry associated with disclosure of research results. They would not expect researchers to disclose results that did not provide utility, since these could only provoke worry.

Cystic Fibrosis Participants-Both biobank and CF participants presumed the clinical validity of research results. However, for CF participants, the utility of the results was not as determinate of whether they should be offered as it was for biobank participants. Because CF participants believed that results offered information about their bodies and their condition, they wanted them. Moreover, because of their belief in the promise of research, they expected that even results that are not useful now could be useful in the future. As one respondent explained:

I mean if they find something like totally unexpected, and they have no idea what it is, I think they should tell a person. ... Maybe they could do more research on the person and maybe actually find out what's causing it. [Interviewer: Are there any problems you can think of with giving people those kinds of individual genetic research results?] No. I don't think there's any problems. I would think it would be a benefit. Then the person will be more informed if something happens later down the road to them. $(\mathrm{C} 02)$

In general, $\mathrm{CF}$ participants were more comfortable than biobank participants with uncertainty. One respondent, asked why he thought researchers should offer individual results with uncertain implications, explained:

Just to tell them, I don't know, "Hey. This might be something that's wrong, but we're not a hundred percent." Or "This might be why you don't do so and so or feel so and so." I think it's good to tell everybody everything. [Interviewer: What do you think the benefit is to those people to find out that information?] To the person probably nothing. Just the fact of knowing. (C16)

Thus CF participants expressed hope in research and saw receiving results-even results with uncertain validity and utility—as informing them about their bodies and condition.

Importantly, the CF participants had an existing, and commonly close, relationship with the researchers-one that was not present for biobank participants. CF participants seemed comfortable relying on the researcher (who was frequently also their physician) to decide when results should appropriately be given. Unlike biobank participants, they also had a strong affinity with a patient community. In the interviews, they acknowledged that they 
participated in studies because they believed the studies may help themselves and/or the " $\mathrm{CF}$ community," and because they trusted and wanted to help their physician - who is often the person asking them to participate in the study. This faith in their physicians translated into faith in them as researchers. As one respondent noted when explaining why she participates in studies, "If [the physician-researchers] approach me for a research study, then I feel like it's for an important cause or an important reason for the condition" (C17).

For many CF participants, attitudes toward receiving individual genetic results from research studies were bound to their feelings about the physicians and researchers at the clinic. For example, one respondent, who said she would always prefer to receive genetic research results, was asked how she felt about not getting individual results. She remarked, "I was okay with that because I figure if there was anything important worth noting, they would have been happy to let me know" (C17). Despite their stated desire to receive meaningful individual research results and a lower threshold for validity and utility, their trust in the physician-researchers led these participants to defer to those professionals' decisions on the return of individual results.

\section{Discussion}

Our findings suggest that these individuals' statuses, as patient-participants with a known genetic disorder or as healthy volunteers without a chronic health condition, informed their conceptualizations of the meaningfulness of results, their opinions about the return of individual genetic research results, and their desires for particular types of research results. We note with interest that virtually no participants recalled receiving genetic information when recruited for the followup study. Their talk of individual research results in the course of the interview was based on their assumptions of what results would or should look like. These views permeated the interviews and seemed to persist despite the definition of individual genetic research results that we gave immediately before asking for their views about individual results. Our definition of individual results was meant to convey the potential ambiguity of research results, and thus it deliberately highlighted that researchers themselves may not understand the meaning of results. Because many of our biobank respondents were themselves researchers, we might expect this definition to be particularly resonant with this group of well-educated and knowledgeable individuals, yet we did not find that their views were shaped by it.

Equally interesting is our finding that almost no one realized they had participated in a genotype-driven study and received genetic information as part of their own recruitment. Even so, our interviews provided rich data about how patient-participants and healthy volunteers perceive disclosure of results with regard to validity, utility, and personal, population, and societal implications.

Though our interview protocol did not specifically ask respondents about their perceptions of meaningfulness of individual genetic research results, the idea that results were meaningful was an important touchstone for participants as they reflected on the content and usefulness of such results. While conceptions of meaningfulness differed between the two groups, it typically invoked some combination of validity and/or utility. Healthy volunteers commonly equated individual genetic results with the disclosure of bad news. Thus, a research result was assumed to be valid (real knowledge) and disclosure would be necessarily tied to "bad news," while lack of disclosure could imply good news. For those participants with a known genetic condition (cystic fibrosis), their desire for information about their bodies and their disease informed their impression that research results would be meaningful. They often assumed that results would be clinically valid and utilizable. However, their belief in the promise of research to help ameliorate the condition for 
themselves or others with cystic fibrosis meant they were more comfortable with uncertain research results. Moreover, unlike biobank participants who assumed results would be bad news, CF participants had hope that results might offer news that would ultimately benefit them such as new therapies or a cure (see Tabor et al., 2011, for a similar result among parents of children with autism).

Second, the meaningfulness assigned to individual genetic results must be understood in the context of when and how disclosure would occur. Many biobank participants viewed disclosure of research results as appropriate whenever they perceived that clinical or personal utility outweighed the worry associated with disclosure of research results. In contrast, despite their stated desire to receive individual research results and their general acceptance of a lower threshold of validity and utility, the CF participants trusted their physician-researchers to decide whether results should be disclosed. It may also be that their deference to professional judgments influenced their own assessments of the meaningfulness of results.

\section{Best Practices}

The data from these interviews provide participant perspectives to inform policy recommendations on genotype-driven research recruitment. Importantly, our study includes two types of research participants who may be targeted for genotype-driven recruitment in the future: patients with a known genetic condition who consented to at least one prior genetic research study, and healthy volunteers for a genetic biobank for whom recontact for additional studies is part of participation in the biobank.

With regard to the issue of whether individual genetic results should be provided as part of that recruitment, our findings are similar to those found at other sites comprising the larger study of genotype-driven recruitment (Beskow et al., 2011). Specifically, respondents advocated a lower threshold for return of individual genetic research than is generally recommended (Beskow et al., 2011). Yet, as the larger study also reported, ideas varied between the different study populations regarding the specific circumstances under which research results should be conveyed as part of that recruitment. These empirical results support Beskow and Burke's (2010) suggestion that, in deciding when to offer individual results, researchers and institutional review boards should consider both the nature of the results (for example, their validity and utility) and the context (for example, the participant population and their relationship with the researcher) in which the research is conducted.

Furthermore, our findings suggest the importance of researchers' awareness of the variety of meanings participants assign to individual genetic research results. These understandings and interpretations can lead to different notions about the validity and utility of individual genetic research results, even among highly educated individuals with significant experience in research contexts. For this reason, we suggest that when researchers do return results as part of the recruitment process, they offer written and verbal explanations that clearly describe the results within the context of likely participant conceptions of meaning. Moreover, we suggest these results be separated from the consent form that participants are asked to sign so that the results are not inadvertently hidden within the form.

\section{Research Agenda}

The contributions of this study lie in data that are drawn from the TGF $\beta 1$ follow-up study's use of patient-participants as well as healthy volunteers. Given our small sample size, and non-random recruitment from a larger number of participants in the TGF $\beta 1$ study, findings of similarity as well as differences must be interpreted with caution. We believe that similar findings, when differences might otherwise have been expected, are quite suggestive, and 
that the striking differences related to patient-participant versus healthy volunteer status merit further investigation.

Special note should be made regarding the recruitment information from the TGF $\beta 1$ study; prospective participants were told about categorical genetic inclusion criteria (e.g., that eligibility was based on having either the TT or CC variant) but were not given their individual results. This type of genotype-driven research recruitment raises additional important research questions. How does provision of aggregate or categorical genetic information compare to the circumstance of return of individual genetic research results? How should researchers approach participants' requests for specific rather than categorical results? In our interviews, most people did not recall being provided categorical research results during recruitment. When we showed respondents the genetic inclusion criteria at the end of the interview, most were confused by this information, and some biobank participants wondered about possible implications for their health or reproductive decision-making. Are there other circumstances in which such genetic inclusion criteria might present more concerns? Are there better ways to offer research results, taking advantage of both research and lay perceptions of utility?

Other important questions remain that should be the subject of future research. For example, do patient-participants with a medical condition without an obvious genetic basis perceive the meaning of results differently than these CF participants who have known about their condition and its genetic basis for most of their lives? Data from our larger study suggest they do (Namey \& Beskow, 2011; Tabor et al., 2011), and merit further exploration.

\section{Educational Implications}

In addition to the implications suggested by the results from our cross-site study (Beskow et al., 2011), it is important to recognize that genotype-driven research recruitment may increasingly target individuals who participate in biobanks or genetic registries. These biobanks will have tremendous variation in the populations they recruit (ranging from disease specific to general population), consent they employ, and oversight regarding access to samples and data. In all cases, it is important for researchers and IRBs to be aware of the various contextual factors associated with the research study and plan their recruitment procedures (including the decision about disclosure of results) accordingly.

\section{Acknowledgments}

We would like to thank Patricia Chulada, PhD, MHS, Health Scientist Administrator from the National Institute of Environmental Health Sciences for her invaluable help associated with the Environmental Polymorphisms Registry. We also thank Michael Knowles, MD, Professor of Pulmonary Medicine in the UNC Department of Medicine and the UNC Cystic Fibrosis Treatment and Research Center, and Cindy Sell, Administrative Support Associate at UNC for their assistance associated with the TGF 1 study. Many thanks also to the CF and biobank participants who agreed to be interviewed. The project described was supported by Award Number RC1HG005787 from the National Human Genome Research Institute (NHGRI). Support was also provided by P50 HG004488 from the NHGRI. The content is solely the responsibility of the authors and does not necessarily represent the official views of NHGRI or the National Institutes of Health.

\section{Biographies}

R. Jean Cadigan is Research Assistant Professor in the Department of Social Medicine at the University of North Carolina-Chapel Hill. She is an anthropologist interested in the ethical, legal, and social implications of genetic research for participants, investigators, and those who manage biobanks. She conducted interviews, led the development of the codebook and data coding, worked on the data analysis for this manuscript, and wrote it in collaboration with the four co-authors. 
Marsha Michie is a Postdoctoral Fellow at the Center for Genomics and Society at the University of North Carolina-Chapel Hill. She is a cultural anthropologist whose research interests include the ethical and social implications of genetics and genomics, genetic identities and moralities, and religion in everyday life. Dr. Michie conducted interviews, participated in the development of the codebook and the coding of data, and collaborated in the analysis of data and the writing and revision of the manuscript.

Gail Henderson is Professor and Chair of the Department of Social Medicine, in the School of Medicine at the University of North Carolina-Chapel Hill, and Director of the UNC Center for Genomics and Society, an NHGRI center, funded to explore ethical, legal, and social implications (ELSI) of the human genome project. She is a sociologist whose research addresses ELSI aspects of large-scale genomic research. She is the UNC-site PI for the present project, and participated in development of the proposal and the interview guides, and provided comments and revisions of the manuscript as it was being written.

Arlene M. Davis is a faculty member in the UNC Department of Social Medicine and the Center for Bioethics. Davis's prior legal research regarding biobank governance and her research regarding participant perspectives on biobank participation informed her work on this project, where she collaborated on creation of the interview guide, analysis, and authorship of this manuscript.

Laura M. Beskow is an Assistant Research Professor at the Duke Institute for Genome Sciences and Policy, where her research focuses on ethics and policy issues in large-scale genomic research and translation. She is Principal Investigator of the larger three-site study exploring participants' perspectives on genotypedriven research recruitment, for which she conceived and led the design of the study, led the development of the interview guide, and oversaw data collection. For this analysis of the UNC site's data, she assisted in the interpretation of the data, made critical revisions for important intellectual content, and approved the final version.

\section{References}

Beskow LM, Burke W. Offering individual genetic research results: Context matters. Science Translational Medicine. 2010; 2(38):38cm20.

Beskow LM, Namey EE, Cadigan RJ, Brazg T, Crouch J, Henderson GE, et al. Research participants' perspectives on genotype-driven research recruitment. Journal of Empirical Research on Human Research Ethics. 2011; 6(4):3-20. [PubMed: 22228056]

Bookman EB, Langehorne AA, Eckfeldt JH, Glass KC, Jarvik GP, Klag M, et al. Reporting genetic results in research studies: Summary and recommendations of an NHLBI working group. American Journal of Medical Genetics Part A. 2006; 140A:1033-1040. [PubMed: 16575896]

Bredenoord AL, Kroes HY, Cuppen E, Parker M, van Delden JJM. Disclosure of individual genetic data to research participants: The debate reconsidered. Trends in Genetics. 2011; 27(2):41-47. [PubMed: 21190750]

Cadigan, RJ.; Davis, AM. Deciding whether to participate in a biobank: The concerns of healthy volunteers. In: Kaye, J.; Stranger, M., editors. Principles and practice in biobank governance. Farnham, UK: Ashgate; 2009. p. 117-133.

Charmaz, K. Constructing grounded theory: A practical guide through qualitative analysis. Thousand Oaks, CA: Sage; 2006.

Chulada PC, Vahdat HL, Sharp RR, DeLozier TC, Watkins PB, Pusek SN, et al. The Environmental Polymorphisms Registry: A DNA resource to study genetic susceptibility loci. Human Genetics. 2008; 123(2):207-214. [PubMed: 18193459]

Cutting GR. Modifier genes in Mendelian disorders: The example of cystic fibrosis. Annals of the New York Academy of Sciences. 2010; 1214:57-69. [PubMed: 21175684] 
Dressler LG. Disclosure of research results from cancer genomic studies: State of the science. Clinical Cancer Research. 2009; 15(13):4270-4276. [PubMed: 19549775]

Drumm ML, Konstan MW, Schluchter MD, Handler A, Pace R, Zou F, et al. Genetic modifiers of lung disease in cystic fibrosis. New England Journal of Medicine. 2005; 353:1443-1453. [PubMed: 16207846]

Fabsitz RR, McGuire A, Sharp RR, Puggal M, Beskow LM, Biesecker LG, et al. Ethical and practical guidelines for reporting genetic research results to study participants: Updated guidelines from a National Heart, Lung, and Blood Institute Working Group. Circulation: Cardiovascular Genetics. 2010; 3:574-580. [PubMed: 21156933]

Fisher R. A closer look: Are we subjects or are we donors? American Journal of Bioethics. 2008; 8(11):49-50. [PubMed: 19061111]

Hoeyer K. Donors perceptions of consent to and feedback from biobank research: Time to acknowledge diversity? Public Health Genomics. 2010; 13(6):345-352. [PubMed: 19940458]

Michie M, Henderson G, Garrett J, Corbie-Smith G. "If I could in a small way help": Motivations for and beliefs about sample donation for genetic research. Journal of Empirical Research on Human Research Ethics. 2011; 6(2):57-70. [PubMed: 21680977]

Miles, MB.; Huberman, AM. Qualitative data analysis: An expanded sourcebook. Thousand Oaks, CA: Sage; 1994.

Miller FA, Christensen R, Giacomini M, Robert JS. Duty to disclose what? Querying the putative obligation to return research results to participants. Journal of Medical Ethics. 2008; 34(3):210213. [PubMed: 18316466]

Miller FA, Hayeems RZ, Bytautas JP. What is a meaningful result? Disclosing the results of genomic research in autism to research participants. European Journal of Human Genetics. 2010; 18(8): 867-871. [PubMed: 20234389]

Murphy J, Scott J, Kaufman D, Geller G, LeRoy L, Hudson K. Public expectations for return of results from large-cohort genetic research. American Journal of Bioethics. 2008; 8(11):36-43. [PubMed: 19061108]

Namey EE, Beskow LM. Epilepsy patient-participants and genetic research results as "answers". Journal of Empirical Research on Human Research Ethics. 2011; 6(4):21-29. [PubMed: 22228057]

National Bioethics Advisory Commission. Research involving human biological materials: Ethical issues and policy guidance. Vol. 1. Rockville, MD: U.S. Government Printing Office; 1999.

NVivo qualitative data analysis software. QSR International Pty Ltd; 2008. Version 8

Tabor HK, Brazg T, Crouch J, Namey EE, Fullerton SM, Beskow LM, Wilfond BS. Parent perspectives on pediatric genetic research and implications for genotype-driven research recruitment. Journal of Empirical Research on Human Research Ethics. 2011; 6(4):XX-XX.

Trinidad SB, Fullerton SM, Ludman EJ, Jarvik GP, Larson EB, Burke W. Research ethics - Research practice and participant preferences: The growing gulf. Science. 2011; 331(6015):287-288. [PubMed: 21252333] 


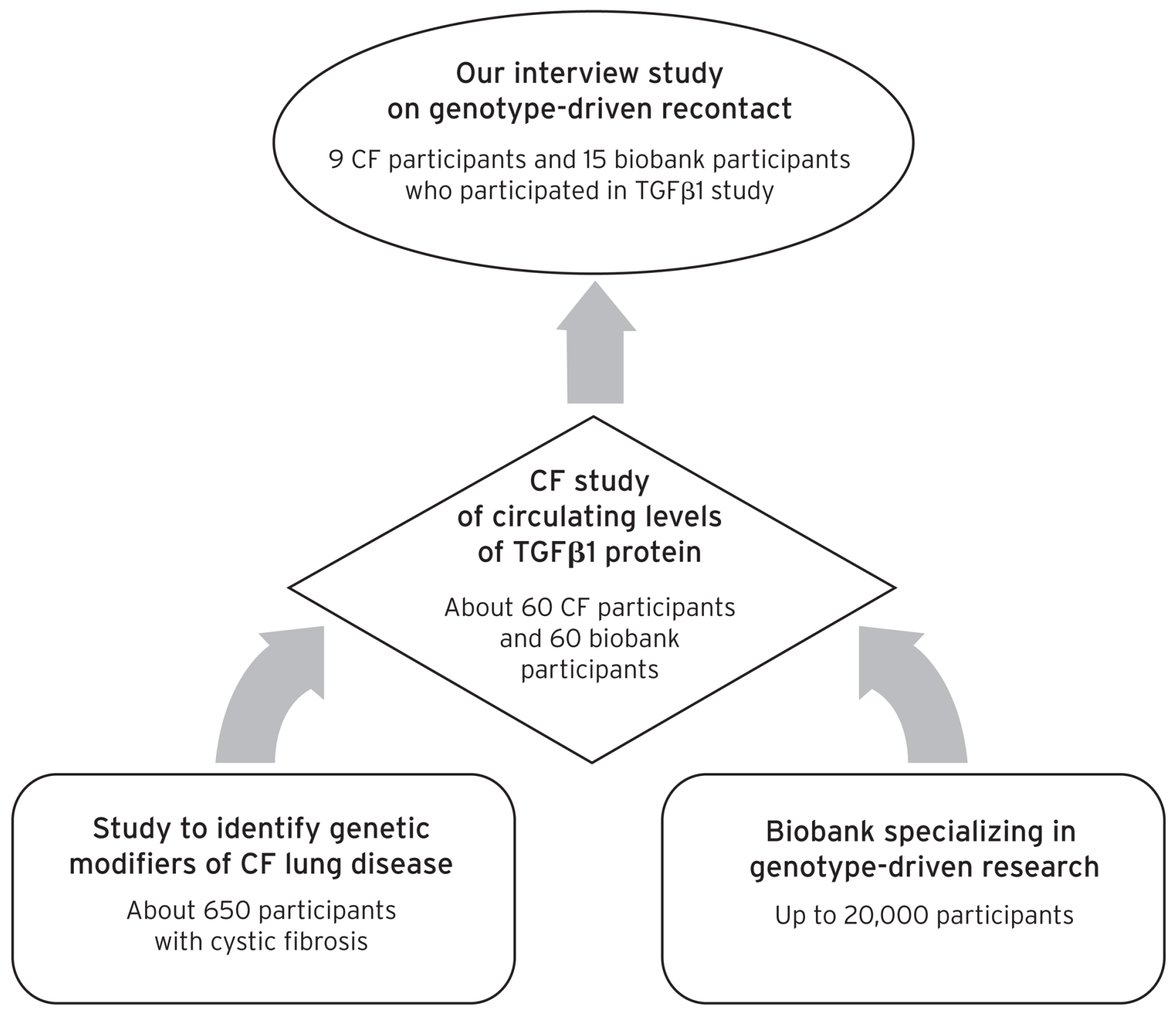

Fig. 1.

Depiction of the Relationship Between the Various Studies. 
Table 1

Respondent Demographics.

\begin{tabular}{lcc}
\hline & Cystic Fibrosis (CF) Participants (N=9) & Biobank Participants (N=15) \\
\hline Age Range (years) & $24-50$ & $25-57$ \\
$\quad$ Mean Age (years) & 32 & 38 \\
Gender & 6 & 9 \\
Male & 3 & 6 \\
Female & & \\
Highest Level of Education & 3 & 0 \\
Some College & 3 & 5 \\
4-Year College Degree & 3 & 10 \\
Graduate/Professional Degree & & \\
\hline
\end{tabular}

